\title{
Patient and clinician engagement with health information in the primary care waiting room: A mixed methods case study
}

\author{
Cara Penry Williams, Kristine Elliott, Jane Gall, Robyn Woodward-Kron \\ University of Melbourne, Australia
}

\begin{abstract}
Significance for public health
Primary care waiting rooms are traditionally sites of health promotion, with leaflets and posters providing opportunities for patient education about disease prevention and treatment information for common illnesses. This case study in regional Australia investigated how and to what extent patients, accompanying persons and primary care providers engaged with the health information in their waiting rooms. Despite the ubiquity of personalised digital communication tools, the findings showed that patients engage with and continue to value health information in the general practice waiting room. By knowing more about patient behaviours and preferences for the mode of health messages (e.g. via screen), medical practices can better target their audience. The study also investigated the connection between the health messages in the waiting room and whether this activated patients to ask their doctor about these messages. To optimise the benefits of patient education materials in the waiting room, practices should seek opportunities to keep clinicians up to date with the resources on offer and refer patients to these resources when relevant as did the practice nurses in this study. We also suggest that clinician familiarity with the resources can be fostered by a dedicated person in the practice. For example, at the conclusion of this case study, the clinic involved decided to add patient health information resources to the weekly meeting agenda, for which one of the registrars volunteered to take responsibility.
\end{abstract}

\section{Abstract}

Background. Primary care waiting rooms can be sites of health promotion and health literacy development through the provision of readily accessible health information. To date, few studies have considered patient engagement with televised health messages in the waiting room, nor have studies investigated whether patients ask their clinicians about this information. The aim of this study was therefore to examine patient (or accompanying person) and clinician engagement with waiting room health information, including televised health messages.

Design and methods. The mixed methods case study was undertaken in a regional general practice in Victoria, Australia, utilising patient questionnaires, waiting room observations, and clinician logbooks and interviews. The qualitative data were analysed by content analysis; the questionnaire data were analysed using descriptive statistics.

Results. Patients engaged with a range of health information in the waiting room and reportedly received health messages from this information. $44 \%$ of the questionnaire respondents (33 of 74) reported watching the television health program, and half of these reported receiving a take home health message from this source. Only one of the clinicians $(N=9)$ recalled a patient asking about the televised health program.

Conclusions. The general practice waiting room remains a site where people engage with the available health information, with a televised health 'infotainment' program receiving most attention from patients. Our study showed that consumption of health information was primarily passive and tended not to activate patient discussions with clinicians. Future studies could investigate any link between the health infotainment program and behaviour change.

\section{Introduction}

A 'healthy' waiting room with provision of health promotion material for patient education, is a concept with a long history in general practice ${ }^{1,2}$ and other primary care settings. ${ }^{3,4}$ There are numerous benefits to having patient education materials available in the waiting room. Firstly, patients waiting for an appointment are a captive audience, ${ }^{5}$ likely to be pre-disposed to engage with health promotional material. Secondly, if aligned with patient-centred care, the availability of health promotion materials can promote general health and contribute to improved patient satisfaction. ${ }^{1,6,7}$ Thirdly, there is evidence of their effectiveness ${ }^{8}$ which holds across formats and topics, including leaflets and posters, ${ }^{4,9}$ and interventions using videos about general health topics, ${ }^{10,11}$ vaccines, ${ }^{12}$ parenting, ${ }^{13}$ and sexual health. ${ }^{14,15}$ Waiting room interventions can engage patients with increased risk who may not normally access health information. ${ }^{16} \mathrm{~A}$ further factor recommending waiting rooms as sites of health promotion is the role general practitioners (GPs) can play in tailoring Internet-sourced health information for patients. ${ }^{17,18}$ Patients are therefore likely to associate their GPs, and by implication the health information their practices provide, as trusted sources.

Health literacy has been traditionally conceptualised as the ability to read and comprehend medical information. More expanded conceptualisations include communicative and critical health literacy, ${ }^{19}$ where communicative health literacy refers to the oral, interactional skills needed to discuss health information with clinicians, and critical health literacy to the ability to analyse health information to order to inform decisions. The U.S. National Institutes of Health's definition extends the concept of health literacy to the "materials, environments, and challenges specifically associated with disease prevention". ${ }^{20}$ These factors as well as people's communicative and critical health literacy impact on people's ability to make decisions about their health, prevent disease, and access healthcare. ${ }^{21}$ Communicative health literacy as well as critical health literacy are also needed to make sense of the plethora of leaflets in primary care, particularly as these materials may provide contradictory messages. ${ }^{22} \mathrm{~A}$ review of interventions in primary care to improve health literacy for chronic disease showed that many different types of interventions (e.g. leaflets, video, group sessions, individual counselling) could facilitate positive change in health literacy. ${ }^{23}$ Different patterns emerged for 
positive change depending on the type of intervention, setting and chronic condition. ${ }^{23}$ Computer-based interventions were not included in the review nor were televised health and well-being programs although there is increasing availability of such programming at point of care.

Despite the potential of the waiting room as a facilitative environment for health promotion and patient education, ${ }^{21,24,25}$ there are few studies that have examined patient or clinician preferences for, and uses of health information from the waiting room, ${ }^{9}$ or studies that have monitored patients' naturalistic interactions with materials, or that have investigated whether patients follow up about waiting room materials with their GP. Understanding these factors is key to designing effective materials. This study investigated patient, accompanying person, and clinician perspectives on and engagement with waiting room health information in a general practice, including a narrowcast television program promoting healthy lifestyle and disease prevention. We use the term engagement to refer to patients' and accompanying persons' observed interactions with the health information as well as reported cognitive aspects such as understanding and awareness raising, and intended future behaviours such as health information seeking. We also explored the role of waiting room materials in the consultation and clinician views on waiting room materials for health promotion.

\section{Design and methods}

A mixed methods case-study design was used to investigate the waiting room as a place for patient education, and to gain insights into the mention or utilisation of waiting room resources in the clinician-patient consultation. ${ }^{26,27}$ A case study design was deemed appropriate to understand the complex and natural setting in detail. ${ }^{28}$ Further, the study was naturalistic in that no interventions were implemented by the researchers. Previous multi-site studies in primary care on patient preferences for health information have used surveys as a data collection tool. ${ }^{9}$ Other topic-focussed studies have employed observations and interviews. ${ }^{29}$ Here, to gain a fuller picture, we applied both quantitative and qualitative approaches: i) a questionnaire to provide a quantitative measure of patient self-report of their waiting room preferences, and practices in regards to health information seeking and potential behaviour change, ii) observation of patient engagement with health information in the waiting room, iii) a clinician logbook of patient enquiries about health information from the waiting room, iv) and semi-structured interviews with clinicians to explore their perspectives on waiting room health information. For a critical discussion of the merits, disadvantages and challenges of using mixed methods, refer to Brannen and O'Connell. ${ }^{30}$

Approval for the study was provided by the University of Melbourne Human Ethics Advisory Committee [1442871].

\section{Context of the study}

This study was conducted in one regional general practice in the state of Victoria, Australia. At the time of the study in 2014, the practice had six full-time GPs, two practice nurses and one social worker (part-time). The practice's commitment to patient education, the area's rural population combined with low-income households and one of the lowest school retention rates in the state recommended it as a study site, as these social factors are associated with low health literacy. ${ }^{21,31}$ A range of health information was available in the large waiting room of the practice. This included a televised health and well-being programming, the Australian-produced Tonic Direct, delivered via a large television screen on the wall near the entrance. Tonic Direct (now rebranded as Tonic on Demand) covers a range of topics, including chronic disease, and lifestyle modifications to promote health in short segments. Many segments are hosts exploring and explaining a topic in journalistic style but there are also less serious segments with people cooking healthy meals and a comedian sharing his thoughts on a medical or lifestyle topic. Directly below the television was a notice board. On and next to the notice board were several posters on disease prevention topics, including men's health, and heart disease. The back wall of the waiting room, which was closest to the consultation rooms, contained a well-ordered display of health information with over 70 pamphlets on a wide range of topics. The proximity to the consultation rooms allowed the practice nurses to access these pamphlets easily, while the distance from the seating provided patients with some privacy in which to browse this source of health information. All the health information was displayed in a tidy, accessible way. In addition, the waiting room contained magazines dispersed on small tables amid the fixed seating. There were no health focussed magazines; instead the magazines included lifestyle magazines targeting women, as well as fishing and motoring magazines. Some of the lifestyle magazines may have included health messages; however, we did not audit the contents of these magazines. The waiting room included a large area with playequipment for children towards the back of the room. The majority of the data were collected during a ten-day period with follow-up interviews conducted after this more intensive period of study.

\section{Instruments, participants and recruitment}

Prior to commencement of the study, an information session was held at the practice to inform management, administration and clinical staff about the study. Study procedures were discussed and subsequently conducted by researchers with practice approval. At the session all clinicians agreed to participate in the study and signed consent forms.

\section{Waiting room questionnaire}

The purpose of the questionnaire was to investigate patient perceptions of their activities while waiting, particularly whether and how they engaged with health information. It included questions on demographic information, their waiting room activities on that day (e.g. reading a magazine, using a mobile device, minding children etc.), the time spent on activities, and whether they acknowledged health messages from various sources. There were also questions about waiting room preferences generally. The questionnaire also included items intended to give an indication of patients' self-reported functional, communicative, and critical health literacy, ${ }^{19,32}$ (I ask the questions I need to ask when I talk to my doctor and/or nurse; I take an active role in managing my health and well-being; I compare information from different sources and then decide what is best for my health). The questionnaire was piloted with five patients and two clinicians from the study practice.

The questionnaire was administered by researchers in the waiting room during two-hour blocks, which were pre-scheduled for different times of the day, over the ten-day period. Waiting patients and accompanying persons over eighteen years of age were approached by a researcher, informed about the study, and given a plain language statement to read and retain. Those who agreed to participate then signed a consent form, before completing the questionnaire. As part of the protocol, it was agreed that patients who were acutely unwell were not to be approached. Reception staff assisted in identifying these patients. 


\section{Waiting room observations}

While one researcher administered the questionnaire, a second researcher made notes on the general ambience of the waiting room and waiting room activities during the two-hour period. This researcher sat in a corner of the waiting room and made notes at 30-minute intervals using an observation form, which noted the date, and observation times. Notes were made under the following sub-headings: activities observed; level of activities; busyness of waiting room, including approximate number of people present; and television viewing. Under television viewing we noted whether people chose to sit facing the screen or with their backs to the screen, whether people watched the television screen with undivided attention or shared their attention between screen, magazines, leaflets, their smartphones or other modalities, which we referred to as browsing in the notes. A second observation form was used to show the layout of the play area, the television screen and the seating. Photos were taken of the waiting room when it was empty in case the set up changed between observations. The four researchers trialled the observation forms and agreed upon terms such as browsing. The observation notes were important for grounding the self-reported individual actions within a larger picture and understanding the waiting room as an 'ecology'. ${ }^{33}$

Patients and accompanying persons were informed of the observations by an information leaflet at the reception desk, and verbally informed by reception staff when they approached the reception desk. Those wishing to be excluded from the observations were asked to notify the reception staff. No patients or accompanying persons asked to be excluded from the observations.

\section{Clinician logbooks}

Over the ten-day period, clinicians were asked to record in a logbook any instance when a patient enquired about external health information, including from a waiting room source. Clinicians also recorded instances when they recommended health information to patients, including from the waiting room, together with the type of information recommended and the topic.

\section{Clinician interviews}

Semi-structured interviews with the clinicians explored their practices, experiences, and perspectives on the use of health information materials at the practice. Interviews were conducted by one researcher (RWK) at the conclusion of the logbook data collection phase. The researcher had been introduced to all clinicians during the information session, but was known professionally to four clinicians prior to the study. Interviewees were asked to comment on their logbook findings and were then asked further questions such as: What is your view on patients getting information on health issues from sources other than medical professionals? What do you think of the waiting room as a place for patient education? What do you know about the Tonic Direct programming? Do you have any further thoughts on the issue of increasing patient engagement in terms of interest and skill in managing their own health? The interviews were audio-recorded and ran for between 10-25 minutes.

\section{Data analysis}

Responses from the waiting room questionnaire were entered into a spreadsheet and the quantitative elements imported into SPSS for simple descriptive statistical analysis. Reported health messages were manually coded according to topic (i.e. Healthy eating, Exercise and fitness, Diabetes, and Smoking), and reported planned behaviour changes were coded by action (e.g. Talking to doctor, Eating better, Exercising more, and Taking more notice of skin changes). Field notes from the waiting room observations were analysed by content and constant comparison methods. ${ }^{34}$ That is, the researchers first read through the field notes individually, then the field notes were compiled according to sub-heading in a spreadsheet. The researchers then compared and contrasted these categories to identify patterns of patient engagement within the waiting room, including how patients interacted with different types of health information. This process involved the researchers individually examining the compiled observations and then coming together to discuss them and reach consensus on identified patterns. Completed logbooks were examined to determine the total number of instances when patients enquired about externally available health information, or when clinicians recommended health information to patients. Recommended health information was coded according to type (e.g. web site, brochure) and broad topic (e.g. preventative health measures, sexual and reproductive health, and mental health). A comparison was made of recommendations by type of health professional (e.g. doctor, nurse, social worker). The notes were confirmed during interviews.

The interview analysis was thematic. Interview transcripts were analysed inductively to identify emerging themes. One researcher (CPW) undertook an initial coding of the data with constant comparison of the nine transcripts. The coding categories were discussed amongst the team and modified until agreement of the major themes was reached. Selected quotes have been used to illustrate the key themes identified.

\section{Results}

The results have been grouped into those methods which investigated patient experiences and perspectives, and those that related to clinician perspectives and experiences of patients asking about health information, including health information in the waiting room.

\section{Patient perspectives}

\section{Waiting room questionnaire}

Seventy-four patients and accompanying persons completed the questionnaire. Their demographic characteristics are shown in Table 1, providing a snapshot of the clinic's clientele during the time of data collection and contextualization of the findings. The majority of respondents was female (69.4\%), aged between either 45-55 years $(26.4 \%)$ or $25-34$ years $(22.2 \%)$, spoke English as their first language (95.8\%), and had a high self-reported level of health literacy based on the questionnaire items (78.1\%). A broad range of occupations was reported: the most frequent were professional $(27.4 \%)$, clerical $(17.4 \%)$, home duties/carers $(15.8 \%)$ (Australian Bureau of Statistics classifications used). Respondents reported waiting between 5 and 60 minutes with the most common response waiting for 20 minutes $(17.6 \%)$. Fewer than 5 people approached declined to participate.

The main findings regarding reported actions are as follows.

Reading or browsing magazines in the waiting room. Almost half of the respondents $(47.3 \%)$ reported reading or browsing magazines while waiting to see a clinician. Of this group of 35,12 reported reading health information about disease prevention. No clear pattern emerged regarding respondents' age, gender, or educational attainment in relation to this activity.

Using a mobile device. Thirty-two (43.2\%) respondents reported using either a smart phone or tablet while waiting. None of these respondents were over 65 years of age. No respondents reported accessing health information on their mobile device. 
Reading leaflets or posters. Eleven participants (14.9\%) reported reading a leaflet or poster. Nine of these were women, six of whom were aged between 45-54 and eight of whom reported having a high level of health literacy. Five women respondents reported receiving take-home health messages. The most common topic was men's health, a focus in the clinic during data collection as part of a national month-long health promotion activity. All 11 respondents anticipated an action or change based on the information, for example, referring their partners to information on men's health.

Watching a televised health and well-being program. Thirtythree participants (44.6\%) reported watching Tonic Direct: 17 of these said that they watched the program for a few minutes, 12 for about 10 minutes and four for more than 20 minutes. There was no significant relationship to collected demographic information about who watched Tonic Direct (based on a chi square analysis). The 33 respondents included 21 women, 11 men and one participant who did not declare a gender. The number of viewers generally decreased with increased age. None of the retired participants reported watching the television $(n=5)$. Tonic Direct viewers represent over half of the participants who reported waiting in excess of 30 minutes (6 of 11). Seventeen viewers reported receiving a take-home health message from Tonic Direct, reporting 24 broad messages, relating to 12 topics. The topics reported by more than two respondents were: healthy eating $(n=6)$, exercise and fitness $(n=4)$, and diabetes $(n=3)$. Eight of these participants suggested they would take some sort of action based on the message: talking to their doctor $(n=2)$, eating better $(n=2)$, exercising more $(n=3)$ and taking more notice of skin changes $(n=1)$.

Based on these data, the television programming provided the most take-home health messages of any medium - just over half of the people who watched the programming reported a take-home message. Table 2 provides a summary of reported waiting room activities and the rate of take-home health messages. Note that while numbers are low, they have been converted to percentages for more direct comparison.

\section{Observations}

Almost 14 hours of observation were completed by three researchers. Patients were observed browsing health information: taking leaflets as well as watching the television programming; they tended to do these activities in a semi-attentive way, simultaneously reacting to stimuli in the waiting room. Certain segments of Tonic Direct seemed to particularly draw in watchers but they often switched back and forth to other activities as segments changed. This was part of the larger phenomenon of 'browsing', perhaps aware that they could be called upon to finish the activity and leave the waiting room at any point. At times the waiting room was reasonably noisy. Some patients sat away from the television, instead facing the clinicians' offices. These patients tended to be older or requiring some form of physical support for walking. Many seemed to want to ensure that they heard their name when called for their appointment (actively waiting).

\section{Clinician perspectives}

\section{Logbooks}

Six of the nine clinicians completed logbooks in the ten-day period, documenting when patients had inquired about external health information, including waiting room information. Five clinicians reported recommending websites to patients, primarily about preventative health measures, men's health, sexual and reproductive health, chronic disease, and mental health. Clinicians also reported referring patients to pamphlets about similar health

Table 1. Demographic characteristics of waiting room questionnaire respondents $(n=74)$.

\begin{tabular}{|c|c|c|c|c|}
\hline Characteristics & & Number & $\%$ & Missing value \\
\hline Gender & $\begin{array}{l}\text { Female } \\
\text { Male }\end{array}$ & $\begin{array}{l}50 \\
22\end{array}$ & $\begin{array}{l}69.4 \\
30.6\end{array}$ & 2 \\
\hline Age & $\begin{array}{l}18-24 \\
25-34 \\
35-44 \\
45-54 \\
55-64 \\
65-74 \\
75-84 \\
85+\end{array}$ & $\begin{array}{c}6 \\
16 \\
10 \\
19 \\
8 \\
10 \\
2 \\
1\end{array}$ & $\begin{array}{c}8.3 \\
22.2 \\
13.9 \\
26.4 \\
11.1 \\
13.9 \\
2.8 \\
1.4 \\
\end{array}$ & 2 \\
\hline English as first language & $\begin{array}{l}\text { Yes } \\
\text { No }\end{array}$ & $\begin{array}{c}69 \\
3\end{array}$ & $\begin{array}{c}95.8 \\
4.2\end{array}$ & 2 \\
\hline Local resident & $\begin{array}{l}\text { Yes } \\
\text { No }\end{array}$ & $\begin{array}{c}65 \\
3\end{array}$ & $\begin{array}{c}95.6 \\
4.4\end{array}$ & 6 \\
\hline Occupation & $\begin{array}{l}\text { Managers \& Administrators } \\
\text { Professionals } \\
\text { Associate professionals } \\
\text { Trades person } \\
\text { Intermediate clerical } \\
\text { Labourers } \\
\text { Retired } \\
\text { Home duties \& Carers } \\
\text { Students } \\
\text { Unemployed }\end{array}$ & $\begin{array}{c}10 \\
19 \\
1 \\
1 \\
12 \\
6 \\
5 \\
11 \\
3 \\
1\end{array}$ & $\begin{array}{c}14.5 \\
27.4 \\
1.4 \\
1.4 \\
17.4 \\
8.7 \\
7.2 \\
15.8 \\
4.3 \\
1.4\end{array}$ & 5 \\
\hline Health literacy & $\begin{array}{l}\text { High } \\
\text { Medium } \\
\text { Low }\end{array}$ & $\begin{array}{c}57 \\
12 \\
4\end{array}$ & $\begin{array}{c}78.1 \\
16.4 \\
5.5\end{array}$ & 1 \\
\hline
\end{tabular}


aspects. The nurses reported recommending health information more frequently than the GPs. Clinicians reported only four instances of patients enquiring about externally available health information. All instances related to patients reporting a website or leaflet that they had found helpful to consult. In the logbook period, no patients asked their clinicians explicitly about Tonic Direct. The findings suggest that during the study period, patients rarely discussed externally available health information with their clinicians.

\section{Interviews}

Expanding on the logbook data, all clinicians reported regularly disseminating health information to patients, such as leaflets and websites, with most reporting that they relied on resources they used frequently and with which they were familiar. Several clinicians mentioned time as a requirement for patient education - time to spend explaining materials to patients, and time to search for and become familiar with available resources. In general, the majority of clinicians felt the waiting room had potential as a site for patient education. To exemplify, one of the nurses commented that she highlighted it as a resource for new patients.

"Any new patients I always say 'there's health information there, you know, please feel free to help yourself while you're waiting for the doctor'," [Nurse 1]

However, a number of concerns were raised regarding that materials should be screened by the clinicians and that patient preferences needed to be taken into account, including health status and privacy:

"There's no privacy, there's just nothing there to support that privacy, and even with a pamphlet, there's limited privacy" [Nurse 1]

The nurse's reference to privacy can be interpreted to mean that patients may wish to retain privacy about their own health status and concerns; subsequently they may not wish to be overheard discussing a pamphlet with a nurse or be seen reading pamphlets about a particular sensitive topic. Technology was noted by some interviewees as a potential remedy to obtaining relevant information without the issues a leaflet can raise.

"the idea of having stuff preloaded on an iPad could be really good, it's more private and discreet" [GP1]

In relation to the then recently installed health 'infotainment' television programming, the clinicians believed that it was a reasonable method of capturing patient attention about health issues and to deliver health information:

"it's a good way to pass on information, because it doesn't entail having to pick up a pamphlet and read it" [Social worker]

The fact that Tonic Direct was utilising a resource that previously had been broadcasting day-time television and advertising or " used to be churning out poor entertainment" [GP2] was seen positively. The varied topics, style of presentation and the fact that it was not tailored for a specific audience, were considered an advantage because it could relate to a broader range of people.

Table 2. Summary of waiting room activities that resulted in reported take-home messages

\begin{tabular}{lccc} 
Activity & $\begin{array}{c}\text { Participants } \\
\text { n }\end{array}$ & $\begin{array}{c}\text { Reported health messages } \\
\text { n }\end{array}$ \\
Tonic Direct TV & 33 & 17 & 51.5 \\
Health leaflet/poster & 11 & 5 & 45.5 \\
\hline Magazine & 35 & 12 & 34.3 \\
\hline
\end{tabular}

\section{Discussion}

Taken together, the results of this study confirm that health information for patient education in the waiting room continues to be utilised by patients and clinicians alike, even in contemporary settings where people can regularly access health information via the Internet to discuss with their clinician or follow up points of interest. ${ }^{17,18}$ The findings from the questionnaire confirm that patients were receptive to the provision of health information in the waiting room. Our study builds on the findings of an existing study 9 by including a televised health awareness program. The televised health infotainment material had the most reports of the available material in terms of promoting awareness raising and intended action or behaviour change. This may be due to the visually appealing format of the information, and the number of health topics covered. Overall, the engagement with health information in the waiting room was impacted by environmental factors such as length of waiting time, general noise in the waiting room, seating arrangements, and the awareness that activities were temporary measures to pass time while waiting for the appointment.

The findings suggest that while the clinicians in this study direct patients to externally available digital and paper-based health information, most of the GPs do so infrequently. For the nurses, the waiting room resources were for incorporating into the closing stage of the consultation when appropriate rather than just for independent patient self-education (confirmed in interviews and by observation). The findings about patient engagement with the health promotional material showed little evidence of transfer from the waiting room to the consultation room: the clinician logbook and interview findings show that no patients followed up with their doctor about health information material from the waiting room during the study period. This finding diverges from findings about patient follow up queries to clinicians about Internetsourced information. ${ }^{17,18}$ One explanation may be that this is due to the defined time period of the logbook data collection compared to previous studies. Activation of patients by waiting room material to 'ask their doctor' may require more exposure over time. We found no evidence in the context of the study that the waiting room materials contributed to developing elements of patients' communicative health literacy (e.g. asking a clinician for related health information); however, it is feasible that patients may have followed up with a health professional or independently sought further information at another point in time. To optimise the benefits of patient education materials in the waiting room, practices should seek opportunities to keep clinicians up to date with the resources on offer and refer patients to these resources when relevant as did the practice nurses in this study. We also suggest that clinician familiarity with the resources can be fostered by a dedicated person in the practice. For example, at the conclusion of this case study, the clinic involved decided to add patient health information resources to the weekly meeting agenda, for which one of the registrar's volunteered to take responsibility.

This study had several limitations. The case study in a regional setting limits the generalisability of these findings. The self-reported health literacy questions showed participants in the study had a mid to high range of health literacy, a finding which surprised the researchers as the setting is considered a low socio-economic area. A follow up study should include a purposive sampling strategy to ensure diversity of health literacy levels as part of a larger sample. It should also include alternative measures to determine health literacy levels. The questionnaire participants did not include nonEnglish speaking background or Indigenous patients and therefore are not representative of the general population. The presence of one or two researchers in the waiting room may have influenced 
people's activity for the observational component; however, with the discrete nature of the observations and the busy waiting room environment, it is likely that the impact of the researchers' presence on the patients' regular waiting room activities was minimal. Several of the data sources depended on participant self-reports; further studies could monitor individual engagement with health information over an extended period in order to examine longitudinal impact of passive health interventions such as waiting room health promotion material. Self-reporting data can be affected by social desirability bias related to participants trying to present themselves well and 'help' researchers. ${ }^{35}$

\section{Conclusions}

This case study suggests that general practice patients and accompanying persons take notice of and engage with health information in the waiting room, including infotainment health programming. Patients in this study engaged with these materials, but they did not follow up about these materials with their clinician(s). Clinicians similarly endorsed health information in the waiting room, acknowledging its potential for patient education. The findings suggest televised health programs can provide patients with health messages, potentially enhancing aspects of health literacy such as knowledge and intended behaviour change. Further research is needed to determine if alternative formats, such as preloaded iPads, are more effective for the promotion of communicative health literacy in the short term. Future studies could also investigate any link between health infotainment programs and behaviour change.

Correspondence: Robyn Woodward-Kron, Department of Medical Education, Melbourne Medical School

University of Melbourne, Parkville VIC 3010, Australia.

Tel.: +61.383443072 .

E-mail: robynwk@unimelb.edu.au

Key words: Health literacy, patient education, general practice, waiting room, health promotion.

Acknowledgments: the authors wish to thank the staff of the participating general practice, and the patient and clinician participants.

Contributions: RWK, KE, JG: conception and design of the study, ethics approval; CPW, RWK, KE: data collection and analysis; CPW, RWK, JG, KE drafting of manuscript, approving final version.

Conflict of interest: the authors declare no potential conflict of interest.

Funding: none.

Conference presentation: Aspects of this paper have been presented at the Royal Australian College of General Practice for General Practice Conference, 2015, Melbourne, Australia.

Received for publication: 18 September 2018.

Revision received: 20 February 2019.

Accepted for publication: 22 February 2019.

(O) Copyright C.P. Williams et al., 2019

Licensee PAGEPress, Italy

Journal of Public Health Research 2019;8:1476

doi:10.4081/jphr.2019.1476

This work is licensed under a Creative Commons Attribution NonCommercial 4.0 License (CC BY-NC 4.0).

\section{References}

1. Blum A. The family physician and health promotion: Do-good- ing or really doing well? Can Fam Physician 1982;28:1613-20.

2. Ward K, Hawthorne K. Do patients read health promotion posters in the waiting room? A study in one general practice. Br J Gen Pract 1994;44:583-5.

3. Taggart J, Williams A, Dennis S, et al. A systematic review of interventions in primary care to improve health literacy for chronic disease behavioral risk factors. BMC Fam Pract 2012;13:49.

4. Protheroe J, Estacio EV, Saidy-Khan S. Patient information materials in general practices and promotion of health literacy: An observational study of their effectiveness. Br J Gen Pract 2015;65:e192-7.

5. Harding C, Hobbs T, Pendrick M. Making use of waiting time: An organisational tool for encouraging health promotion in rural general practice. Aust Fam Physician 2005;34:703-4.

6. Sherwin HN, McKeown M, Evans MF, Bhattacharyya OK. The waiting room "wait": From annoyance to opportunity. Can Fam Physician 2013;59:479-81.

7. Gignon M, Idris H, Manaouil C, Ganry O. The waiting room: Vector for health education? The general practitioner's point of view. BMC Res Notes 2012;5:511.

8. Cass SJ, Ball LE, Leveritt MD. Passive interventions in primary healthcare waiting rooms are effective in promoting healthy lifestyle behaviours: An integrative review. Aust J Prim Health 2016;22:198-210.

9. Moerenhout T, Borgermans L, Schol S, et al. Patient health information materials in waiting rooms of family physicians: Do patients care? Patient Prefer Adher 2013;7:489-97.

10. Koperski M. Health education using video recordings in a general practice waiting area: An evaluation. J R Coll Gen Pract 1989;39:328-30.

11. Cockington R. Health promotion using television in hospital waiting rooms: The Adelaide Children's Parent Education Project. J Paediatr Child Health 1995;31:523-6.

12. Eubelen C, Brendel F, Belche J-L, et al. Effect of an audiovisual message for tetanus booster vaccination broadcast in the waiting room. BMC Fam Pract. 2011;12:104.

13. Kleemeier CP, Hazzard AP. Videotaped parent education in pediatric waiting rooms. Patient Educ Couns 1984;6:122-4.

14. Myint UA, Bull S, Greenwood GL, et al. Safe in the City: Developing an effective video-based intervention for STD clinic waiting rooms. Health Promot Pract 2010;11:408-17.

15. Warner L, Klausner JD, Rietmeijer CA, et al. Effect of a brief video intervention on incident infection among patients attending sexually transmitted disease clinics. PLoS Med 2008;5:e135.

16. Bayer AM, Nussbaum L, Cabrera L, Paz-Soldan VA. Missed opportunities for health education on Pap smears in Peru. Health Educ Behav 2011;38:198-209.

17. Ahluwalia S, Murray E, Stevenson F, et al. 'A heartbeat moment': Qualitative study of GP views of patients bringing health information from the internet to a consultation. Br J Gen Pract 2010;60:88-94.

18. Woodward-Kron R, Connor M, Schulz P, Elliott K. Educating for the patient for healthcare communication in the age of the world wide web: A qualitative study. Acad Med 2014;89.

19. Nutbeam D. Health literacy as a public health goal: A challenge for contemporary health education and communication strategies into the $21 \mathrm{st}$ century. Health Promot Int 2000;15:259-67.

20. National Institutes of Health [Internet]. Clear communication: U.S. Department of Health and Human Services; n.d [Available from: https://www.nih.gov/institutes-nih/nih-officedirector/office-communications-public-liaison/clear- 
communication.

21. Australian Commission on Safety and Quality in Health Care. Consumers, the health system and health literacy: Taking action to improve safety and quality Sydney: ACSQHC; 2013.

22. McCartney M. Patient information leaflets:" a stupid system". BMJ 2013;347.

23. Taggart J, Williams A, Dennis S, et al. A systematic review of interventions in primary care to improve health literacy for chronic disease behavioral risk factors. BMC Fam Pract 2012;13:49.

24. Hill S. Report of the Victorian 2014 consultation on health literacy. Melbourne: Centre for Health Communication and Participation, La Trobe University; 2014.

25. Nouri S, Rudd R. Health literacy in the "oral exchange": An important element of patient-provider communication. Patient Educ Couns 2015;98:565-71.

26. Hunter A, Brewer JD. Designing multimethod research. In: Hesse-Biber S, Johnson RB, editors. The Oxford handbook of multimethod and mixed methods research inquiry. New York; Oxford: Oxford University Press; 2015.

27. Yin RK. Case study research: Design and methods. 5th ed. Los Angeles: SAGE; 2014.

28. Yin RK. Case study research: Design and methods. 4th ed. ed. Thousand Oaks, CA: SAGE; 2009.

29. Rodger D, Skuse A, Wilmore M. 'Passing time': A qualitative study of health promotion practices in an antenatal clinic waiting room. Midwifery, Childbirth and the Media: Springer; 2017. p. 79-96.

30. Brannen J, O'Connell R. Data Analysis 1: Overview of data analysis strategies. In: Hesse-Biber S, Johnson RB, editors. The Oxford handbook of multimethod and mixed methods research inquiry. New York; Oxford: Oxford University Press; 2015. p. 257-74.

31. Australian Bureau of Statistics. Health literacy Canberra: Australian Bureau of Statistics No. 4102.0; 2009 [Available $\mathrm{f} \quad \mathrm{r} \quad \mathrm{o} \quad \mathrm{m}$ : http://www.abs.gov.au/AUSSTATS/abs@.nsf/Lookup/4102.0 Main+Features20June+2009.

32. Ishikawa H, Takeuchi T, Yano E. Measuring functional, communicative, and critical health literacy among diabetic patients. Diabetes Care 2008;31:874-9.

33. Becker F, Douglass S. The ecology of the patient visit: physical attractiveness, waiting times, and perceived quality of care. J Ambul Care Manage 2008;31:128-41.

34. Boeije H. A purposeful approach to the constant comparative method in the analysis of qualitative interviews. Qual Quant 2002;36:391-409.

35. Nederhof AJ. Methods of coping with social desirability bias: A review. Eur J Soc Psychol 1985;15:263-80. 